\title{
Ege Denizi İçin Eşkina Balığı’nın (Sciaena umbra Linnaeus, 1758) Maksimum Boy Kaydı
}

\author{
Özgür CENGIZ ${ }^{1}$ (D), Bayram KIZILKAYA 2(D), Şükrü Şenol PARUĞ 3 (D) \\ ${ }^{1}$ Van Yüzüncü Yıl Üniversitesi, Su Ürünleri Fakültesi, Van, ${ }^{2}$ Çanakkale Onsekiz Mart Üniversitesi, Deniz Bilimleri ve Teknolojisi Fakültesi, \\ Çanakkale, ${ }^{3}$ Kastamonu Üniversitesi, Su Ürünleri Fakültesi, Kastamonu, Türkiye \\ 1https://orcid.org/0000-0003-1863-3482, ${ }^{2}$ https://orcid.org/0000-0002-3916-3734, ${ }^{3}$ https://orcid.org/0000-0002-7991-4651 \\ 凶: ozgurcengiz17@gmail.com
}

\section{ÖZET}

$\mathrm{Bu}$ çalışma Ocak 2013-Aralık 2013 tarihleri arasında Saros Körfezi'nde yürütülmüştür. Maksimum boya sahip bir adet eşkina balığı (41.7 cm toplam boy ve 1100.00 gr toplam ağırlık) 20 Nisan 2013 tarihinde balıkçılar tarafından İbrice Limanı (Saros Körfezi) açıklarında olta ile yakalanmıştır. Tüm bireyler için ağırlık-boy ilişkisi $\mathrm{W}=0.0268 \mathrm{TL}^{2.79}$ olarak hesaplanmıştır. Bu çalışmanın amacı, türün Ege Denizi için maksimum boy kaydını sunmaktır.

Kısa Not
Makale Tarihçesi
Geliş Tarihi : 21.01 .2019
Kabul Tarihi : 09.03 .2019
Anahtar Kelimeler
Sciaena umbra
Eşkina
Maksimum boy
Saros Bay
Ege Denizi

\section{Maximum Size Record of Brown Meagre (Sciaena umbra Linnaeus, 1758) for Aegean Sea}

\begin{abstract}
This study was conducted between January 2013 and December 2013 in Saros Bay. A single specimen of $S$. umbra with the maximum size (41.7 $\mathrm{cm}$ in total length and $180.00 \mathrm{~g}$ in total length) was caught from İbrice Bight (Saros Bay) with hand line by fisherman on 20 April 2013. The weight-length relationship was calculated as $\mathrm{W}=0.0268 \mathrm{TL}^{2.79}$ for all samples. The aim of this study is to present the maximum size record of the species for the Aegean Sea.
\end{abstract}

\section{Short Communication}

$\begin{array}{ll}\text { Article History } & \\ \text { Received } & : 21.01 .2019 \\ \text { Accepted } & : 09.03 .2019\end{array}$

Keywords
Sciaena umbra
Brown meagre
Maximum size
Saros Bay
Aegean Sea

To Cite : Cengiz Ö, Kızılkaya B, Paruğ ŞŞ 2019. Ege Denizi İçin Eşkina Balığı'nın (Sciaena umbra Linnaeus, 1758) Maksimum Boy Kaydı. KSÜ Tarım ve Doğa Derg 22(4): 659-663. DOI: 10.18016/ksutarimdoga.vi.515704

\section{GİRISS}

Sciaenidae familyası, Akdeniz havzasında beş farklı tür ile temsil edilmektedir. Bu türlerden biri olan eşkina balığı (Sciaena umbra Linnaeus, 1758) Azak Denizinden Kanarya Adalarına kadar uzanan geniş bir coğrafyada dağılım gösterir (La Mesa ve ark., 2008). Sürüler halinde yaşayan bu tür çoğunlukla Posidonia yataklarında veya dip yapısı taşlı olan kıyıya yakın yerlerde bulunmaktadır (Fischer ve ark., 1987).

Maksimum boy ve ağırlık değerleri, balıkçılık yönetimi açısından oldukça önemli parametrelerdir (Dulčić ve Soldo, 2005). Bu parametreler, doğrudan ya da dolaylı olarak, stok tayini çalışmalarının çoğunda kullanıldıkları için, bilhassa, ticari olarak sömürülen balık türlerinde maksimum boy ve ağırlık değerlerinin güncellenmesi her geçen gün önem kazanmaktadır (Borges, 2001; Cengiz, 2014).

Bununla beraber, balıkçılık yönetiminde sıklıkla kullanılan diğer bir parametre ise ağırlık-boy ilişkisidir (Erzini, 1994). Bu sebepten dolayı, dünyanın farklı bölgelerindeki balık türlerinin ağırlık-boy ilişkisine değinen yayınların sayısında bir artış söz konusudur (Froese ve ark., 2011). Ağırlık-boy ilişkisi (a) farklı habitatlardan ve/veya bölgelerden yakalanan balık türlerinin yaşam döngülerini ve morfolojilerini kıyaslamaya (Gonçalves ve ark., 1997; Moutopoulos ve Stergiou, 2002), (b) balık türlerinin büyümesindeki mevsimsel değişiklikleri ve kondisyon indekslerini izlemeye (Safran, 1992; Richter ve ark., 2000), (c) bir balık populasyonunun biyokütlesini hesaplamaya (Petrakis ve Stergiou, 1995; Dulčić ve Kraljević, 1996) olanak sağlamaktadır. Bu ilişkiler çalışma bölgesindeki çevresel şartlara ve örnekleme zamanına bağlı olarak değişebileceğinden dolayı (Torres ve ark., 2012) ağırlık-boy ilişkisine yönelik çalışmalar balık populasyonları için düzenli aralıklarla güncellenmelidir (İşmen ve ark., 2007; Özekinci ve ark., 2009).

Türkiyede, balık türlerinin maksimum boy ve ağırlık 
değerlerine yönelik kanıtlanabilir çalışmalar son derece yenidir. Şu ana kadar bu araştırmalar barbun (Mullus barbatus Linnaeus, 1758) (Filiz, 2011), lüfer (Pomatomus saltatrix Linnaeus, 1766) (Cengiz, 2014), gelincik (Phycis phycis Linnaeus, 1766) (Filiz ve Sevingel, 2014), papağan (Sparisoma cretense Linnaeus, 1758) (Filiz ve Sevingel, 2015), kırlangıç (Chelidonichthys lucerna Linnaeus, 1758) (Hasimoğlu ve ark., 2016), çipura (Sparus aurata Linnaeus, 1758 (Aydın, 2018), dil (Solea solea Linnaeus, 1758) (Cengiz, 2018a), iskatari (Spondyliosoma cantharus Linnaeus, 1758) (Cengiz, 2018b) ve zargana (Belone belone Linnaeus, 1761) (Acarli ve ark., 2018) balıkları üzerine yürütülmüştür. Bu çalışma eşkina balığının ağırlıkboy ilişkisini güncellemekle beraber Ege Denizi için türün maksimum boy kaydını sunuyor.

\section{MATERYAL ve YÖNTEM}

Ege Denizi'nin kuzeyinde yer alan Saros Körfezi, güneyde Gelibolu Yarımadası ile kuzeyde Trakya kıyıları arasında bulunan ve yaklaşık olarak $60 \mathrm{~km}$ kadar içeri sokulan üçgen şeklinde bir körfezdir (Tokat ve Sayın, 2007). 2000 yılından beri trol avcılığına kapalı olan körfez (Cengiz ve ark., 2011) 22.12.2010 tarihinde Özel Çevre Koruma bölgesi olarak ilan edilmiştir. Balık örnekleri bölgede Ocak 2013 - Aralık 2013 döneminde balıkçılar yardımıyla 0-30 m arası derinliklerde oltalar ile yakalanmıştır. Örneklerin boyları $\pm 1 \mathrm{~mm}$, vücut ağırlıkları $\pm 0.01 \mathrm{~g}$ hassasiyette ölçülmüştür.

Maksimum boya sahip birey, 20 Nisan 2013 tarihinde İbrice Limanı (Saros Körfezi) açıklarında elde edilmiş, Mater ve ark. (2009) göre tanımlanmış ve bilimsel ismi FishBase'de (Froese ve Pauly, 2018) kontrol edilmiştir. Gıda Tarım ve Hayvancılık Bakanlığı'nın 4/1 Numaralı Ticari Amaçlı Su Ürünleri Avcılığının Düzenlenmesi Hakkında Tebliğ (No： 2016/35)'de toplam boy "ağız kapalı iken balık başının ön ucu ile kuyruk yüzgecinin en uzun ışınının bitim noktası arasındaki izdüşüm uzunluğu" şeklinde ifade edilir.

Türün ağırlık-boy ilişkisi Ricker (1975) tarafından önerilen $W=a L^{b}$ denklemi kullanılmak suretiyle tüm bireyler için hesaplanmıştır. Bu denklemde $W$, gram cinsinden balığın toplam ağırlığı; $L$, toplam boy $(\mathrm{cm}) ; a$ ve $b$ büyümeyi ifade eden sabitlerdir. Eğer $b$ değeri 3 'ten büyük ise tür pozitif allometrik büyüme, 3'den küçük ise negatif allometrik büyüme, 3'e eşit ise izometrik büyüme göstermektedir (Bagenel ve Tesch, 1978). $a$ ve $b$ parametrelerinin sonuçlarına t-testi uygulamak suretiyle $b$ değerinin izometrik büyümeden $(b=3)$ farklı olup olmadiğ ve farklı ise büyümenin pozitif veya negatif olduğuna karar verilmiştir (Avşar, 2005).

\section{BULGULAR}

Bir yıllık çalışma sonunda toplam 14 birey oltalar ile avlanmıştır. Maksimum boy ve ağırlığa sahip bir adet eşkina balığ ise ( $41.7 \mathrm{~cm}$ toplam boy ve $1100.00 \mathrm{gr}$ toplam ağırlık) (Şekil 1) 20 Nisan 2013 tarihinde balıkçılar tarafından İbrice Limanı (Saros Körfezi) açıklarında (Şekil 2) yakalanmıştır.

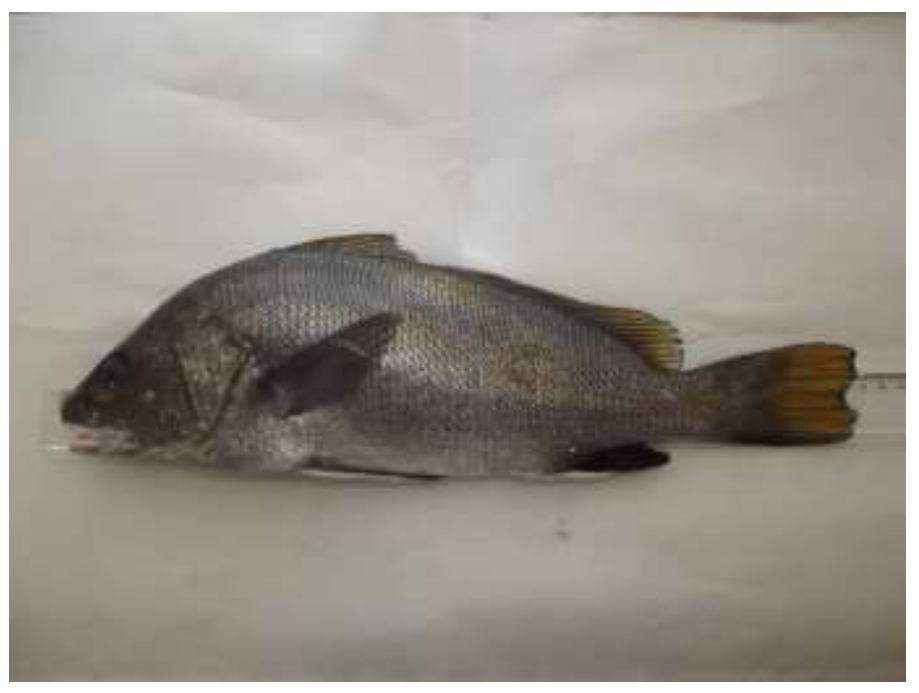

Şekil $1.41 .7 \mathrm{~cm}$ toplam boya ve 1100.00 gr ağırlığa sahip eşkina balığı.

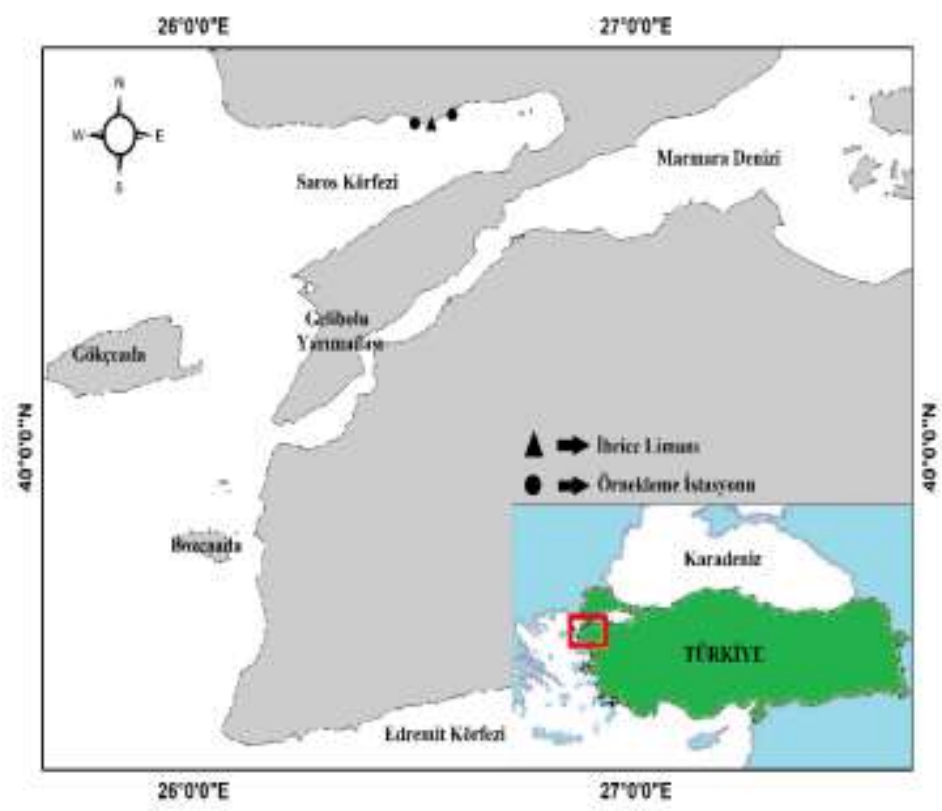

Şekil 2. İbrice limanı ve Örnekleme istasyonu.

Yakalanan balıkların minimum, maksimum boy ve ağırlık değerleri, sırasıyla, $26.0 \mathrm{~cm}$ (300.00 gr) ve 41.7 cm (1100.00 gr)'dir. Bireylerin ortalama boyu ve ağırlığ $34.8 \pm 0.75 \mathrm{~cm}$ ve $635.92 \pm 0.22 \mathrm{~g}$ olarak bulunmuştur. Tüm bireyler için ağırlık-boy ilişsisi W $=0.0268 \mathrm{TL}^{2.79}$ olarak hesaplanmıştır. Eşkina balığının negatif allometrik büyüme gösterdiği saptanmıştır (Şekil 3).

Ege Denizi için eşkina balığının boy ve ağırlık değerlerinin karşılaştırılması ise Çizelge 1'de gösterilmektedir. 
Çizelge 1. Ege Denizi için eşkina balığının boy ve ağırlık değerlerinin karşılaştırılması

\begin{tabular}{lllcccc}
\hline Yazarlar & Bölge & N & $\mathbf{L}_{\text {min }}-\mathbf{L}_{\text {mak }}(\mathbf{c m})$ & W $_{\text {mak }}$ (gr) & a & b \\
\hline Karakulak ve ark. (2006)a & Gökçeada & 24 & $13.9-29.8$ & - & 0.0055 & 3.23 \\
Karachle ve Stergiou (2008) & Thermaikos Körfezi & 11 & $12.2-16.0$ & - & 0.0242 & 2.70 \\
Bilge ve ark. (2014)a & Güney Ege & 54 & $14.7-40.4$ & - & 0.0136 & 3.00 \\
Bu çalışma & Saros Körfezi & 14 & $26.0-41.7$ & 1100.00 & 0.0268 & 2.79 \\
\hline
\end{tabular}

aAkyol ve ark. (2017)

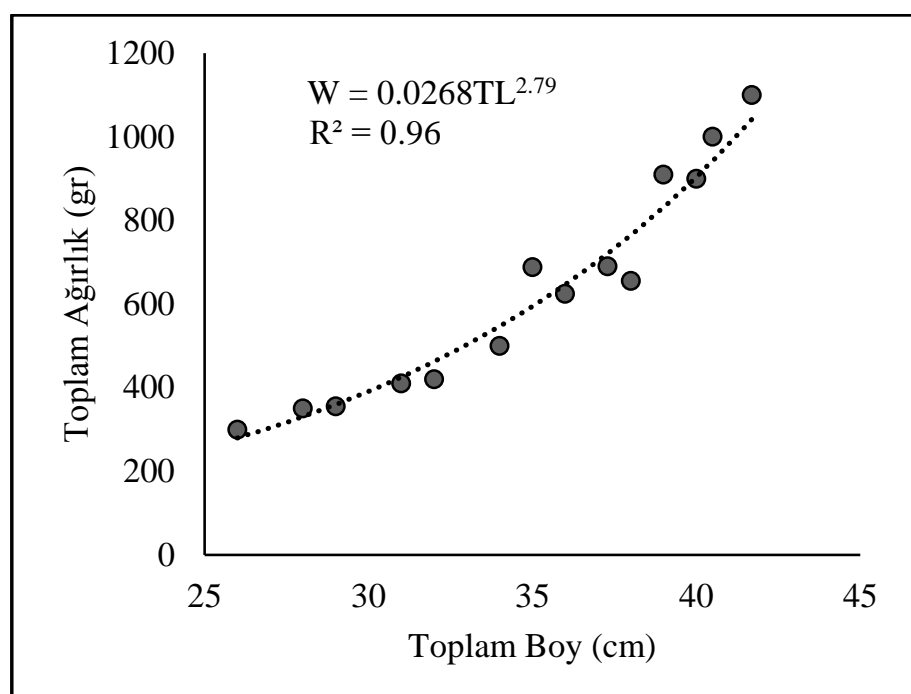

Şekil 3. Eşkina balığının ağırlık-boy ilişkisi

\section{TARTIŞMA ve SONUÇ}

Eşkina balığının dünyada maksimum boyu $70.0 \mathrm{~cm}$ 'dir (Froese ve Pauly, 2018). Bilindiği üzere, aşırı avcılığa maruz kalan türlerin boyları zaman içersinde gitgide küçülmektedir. Eğer bir birey hiçbir av baskısına maruz kalmazsa, maksimum boya ulaşabilir (Filiz, 2011; Cengiz, 2018a).

İlave olarak, balıkların beslenme faaliyetleri ve buna bağlı olarak ortamdaki besin bolluğu; sıcaklık, oksijen, tuzluluk, kirlilik gibi parametre değerleri; predatörlerin varlığı ve türler arasındaki av-avcı ilişkisinin rolü bu çeşit boya ulaşmayı etkileyen diğer önemli unsurlardır (Helfman ve ark., 2009; Acarli ve ark., 2018). Bu yorumlardan yola çıkılarak maksimum boy ve ağırlık değerlerinde görülen bölgesel farklılıkların, aşırı av baskısına ve çevresel şartlara bağlı olduğu sonucu ortaya çıkmaktadır.

Birçok araştırmacı (Özaydın ve ark., 2000; İşmen ve ark., 2007; İlkyaz ve ark., 2010; Kapiris ve Klaoudaos, 2011; Cengiz, 2013) farklı balık türleri üzerinde yaptıkları çalışmalarda boy ve ağırlık aralıklarını etkileyen faktörlerin örnekleme yöntemi ve zamanı, bölgeler arasında görülen sıcaklık varyasyonları, beslenme faaliyetleri, örneklemede kullanılan farklı av araçları ve bu av araçlarının seçicilik özellikleri olduğunu belirtmişlerdir. Benzer şekilde, ağırlık-boy ilişkisi balıkların cinsiyetine, gonad gelişimine, çevresel faktörlerdeki yıllık değiş̧imlere, mevsimsel farklılıklara ve türlerin korunmasına yönelik alınan önlemlere (Dulčić ve Kraljević, 1996; Wootton, 1998; Froese, 2006; Verdiell-Cubedo ve ark., 2006), yakalanan birey sayısına ve bireylerin boy ve ağırlık aralıklarına (Moutopoulos ve Stergiou, 2002) bağlıdır. Çizelge 1'e bakıldığında bu çalışma ile önceki çalışmalar arasında boy ve ağırlık aralıklarında ve ağırlık-boy ilişkilerinde görülün farklılıklar yukarıda bahsedilen bir veya birden fazla etmenle açıklanabilir.

Sonuç olarak, balık stoklarıyla ilgili yapılacak çalışmalardan daha sağlıklı sonuçlar alınabilmesi için maksimum boy ve ağırlık değerleriyle beraber ağırlıkboy ilişkisinin her balık türü için ayrı ayrı gerçekleştirilmesi ve belli zaman dilimleri içerisinde güncellenmesi önem taşımaktadır. Böylelikle bu bilgiler ışığında elde edilecek bulgular, stok ile ilgili mevcut durumu daha net ortaya koyabilir. Bu durum ise, balıkçılık yönetiminde planlanan stratejilerin daha sağlam temeller üzerinde inşa edilmesine olanak sağlar. Bu açıdan bakıldığında, dünyada da her geçen gün sayısı artan böylesi kanıtlanabilir araştırmaların (Borges, 2001; Dulčić ve Soldo, 2005, 2006; Agüero ve ark., 2010; Hossain, 2010; Navarro ve ark, 2012; Hossain ve ark., 2017) Türkiye'de de desteklenmesi sürdürülebilir bir balıkçılık anlayışı için son derece hayati önem taşımaktadır.

\section{TEŞEKKÜR}

Yazarlar yardımlarından dolayı balıkçı Engin Tunç'a ve öğretim görevlisi Semih Kale’ye teşekkür ediyor.

\section{KAYNAKLAR}

Acarlı D, Kale S, Çakır K 2018. A New Maximum Length for the Garfish, Belone belone (Linnaeus, 1761) in the Coast of Gökçeada Island (Aegean Sea, Turkey). Cahiers de Biologie Marine, 59: 385-389.

Agüreo JDLC, Gomez VMC, Navarro JTN 2010. New Maximum Size Record for the Chili Sea Catfish Notarius troschelii (Siluriformes: Ariidae) from the Tropical Eastern Pacific. Marine Biodiversity Record, 3: e107.

Akyol O, Demir Sağlam Y, Ceyhan T 2017. A Collected Work on Length-Weight Relationships of Fish Species in the Aegean Sea. Ege Journal of Fisheries and Aquatic Sciences, 34(2): 235-247.

Avşar D 2005. Balıkçılık Biyolojisi ve Populasyon Dinamiği. Nobel Kitabevi, Adana. 
Aydin M 2018. Maximum Length and Age Report of Sparus aurata (Linnaeus, 1758) in the Black Sea. Journal of Applied Ichthyology, 34: 964-966.

Bagenal TB, Tesch FW 1978. Age and growth. In: Methods for assessment of fish production in fresh waters, (T.B. Bagenal ed), 3rd edition. IBP Handbook No. 3, Blackwell Science Publications, Oxford: 101-136.

Bilge G, Yapıcı S, Filiz H, Cerim H 2014. WeightLength Relations for 103 Fish Species from the Southern Aegean Sea, Turkey. Acta Ichthyologica et Piscatoria, 44: 263-269.

Borges L 2001. A New Maximum Length for the Snipefish Macrohamphosus scolopax. Cybium, 25: 191-192.

Cengiz Ö, İşmen A, Özekinci U, Öztekin A 2011. Saroz Körfezi (Kuzey Ege Denizi) Balık Faunası Üzerine Bir Araştırma. Afyon Kocatepe Üniversitesi Fen Bilimleri Dergisi, 11: 31-37.

Cengiz Ö 2013. Length-Weight Relationships of 22 Fish Species from the Gallipoli Peninsula and Dardanelles (northeastern Mediterranean, Turkey). Turkish Journal of Zoology, 37: 419-422.

Cengiz Ö 2014. A New Maximum Length Record of the Bluefish (Pomatomus saltatrix Linnaeus, 1766) for Turkey Seas. Bitlis Eren Üniversitesi Fen Bilimleri Dergisi, 3: 113-116.

Cengiz Ö 2018a. Weight-Length Relationship with Maximum Size Record of the Common Sole (Solea solea Linnaeus, 1758) in the Aegean Sea. Gece Kitaplığı Yayınevi, Birinci Baskı, Aralık 2018, Ankara, Türkiye, 7-17 s.

Cengiz Ö 2018b. Türkiye Denizleri İçin İskatari Balığı'nın (Spondyliosoma cantharus Linnaeus, 1758) Maksimum Boy Kaydı. International Eurasian Conference on Biological and Chemical Sciences, 26-27April 2018, Ankara, Turkey.

Dulčić J, Kraljević M 1996. Weight-Length Relationships for 40 fish species in the Eastern Adriatic (Croatian waters). Fisheries Research, 28: 243-251.

Dulčić J, Soldo A 2005. A New Maximum Length for the Grey Triggerfish, Balistes capriscus Gmelin, 1789 (Pisces: Balistidae) from the Adriatic Sea. Institute of Oceanography and Fisheries-Split Croatia, 88: 1-7.

Dulčić J, Soldo A 2006. A New Maximum Length for the Garpike Belone belone (Belonidae). Cybium, 30: 382.

Erzini K 1994. An Empirical Study of Variability in Length at Age of Marine Fishes. Journal of Applied Ichthyology, 10: 17-41

Filiz H 2011. A New Maximum Length for the Red Mullet, Mullus barbatus Linnaeus, 1758. Biyoloji Bilimleri Araştırma Dergisi, 4: 131-135.

Filiz H, Sevingel N 2014. A New Maximum Length for the Forkbeard, Phycis phycis (Linnaeus, 1766) in the Mediterranean Sea. Karadeniz Fen Bilimleri
Dergisi, 4: 43-48.

Filiz H, Sevingel N 2015. A New Maximum Length for the Parrotfish, Sparisoma cretense (Linnaeus, 1758) in the Mediterranean Sea. Journal of Aquaculture Engineering and Fisheries Research, 1: 140-143.

Fischer W, Bauchot ML, Schneider M 1987. Fiches FAO d'identification pour les besoins de la pêche révision 1. Méditerranée et mer Noire. Zone de pêche 37, vol. 2: Vertébrés, Rome, FAO, pp. 7611530.

Froese R 2006. Cubelaw, Condition Factor and WeightLength Relationships: History, Meta-analysis and Recommendations. Journal of Applied Ichthyology, 22: 241-253.

Froese R, Tsikliras AC, Stergiou KI 2011. Editorial Note on Weight-Length Relations of Fishes. Acta Ichthyologica et Piscatoria, 41: 261-263.

Froese R, Pauly D (Eds) 2018. FishBase. World Wide Web electronic publication. http://www.fishbase.se /summary/Sciaena-umbra.html (Erişim tarihi: 21.01.2019).

Gonçalves JMS, Bentes L, Lino PG, Ribeiro J, Canário AVM, Erzini K 1997. Weight-Length Relationships for Selected Fish Species of the Small-Scale Demersal Fisheries of the South and Southwest Coast of Portugal. Fisheries Research, 30: 253-256

Hasimoğlu A, Ak O, Kasapoğlu N, Atılgan E 2016. New Maximum Length Report of Chelidonichthys lucerna (Linneaus, 1758) in the Black Sea, Turkey. Journal of the Black Sea / Mediterranean Environment, 22: 149-154.

Helfman GS, Collatte BB, Facey DE, Bowen BW 2009. The Diversity of Fishes: Biology. Evolution and Ecology. 2nd edn. Wiley-Blackwell. UK. 720 p.

Hossain MY 2010. New Maximum Size Record for the Goonch Bagarius yarrelli (Sykes 1839) (Siluriformes: Sisoridae) from the Ganges River. Sains Malaysiana, 39: 157-159.

Hossain MY, Hossen MA, Nawer F, Khatun D, Pramanik MNU, Parvin MF, Yahya K 2017. New Maximum Size Records and Length-Weight Relationships for Two Species, Corica soborna (Hamilton, 1822) and Mystus bleekeri (Day, 1877), from the Ganges River (NW Bangladesh). Journal of Applied Ichthyology, 33: 661-662.

İlkyaz AT, Metin G, Soykan O, Kınacıgil HT 2010. Age, Growth and Sexual Development of solenette, Buglossidium luteum (Risso, 1810), in the Central Aegean Sea. Journal of Applied Ichthyology, 26: 436-440.

İşmen A, Özen O, Altınağaç U, Özekinci U, Ayaz A 2007. Weight-Length Relationships of 63 Fish Species in Saros Bay, Turkey. Journal of Applied Ichthyology, 23: 707-708.

Kapiris K, Klaoudatos D 2011. Length-Weight Relationships for 21 Fish Species Caught in the Argolikos Gulf (central Aegean Sea, eastern 
Mediterranean). Turkish Journal of Zoology, 35: 717-723.

Karachle KP, Stergiou KI 2008. Length-Length and Length-Weight Relationships of Several Fish Species from the North Aegean Sea (Greece). Journal of Biological Research-Thessaloniki, 10: 149-157.

Karakulak FS, Erk H, Bilgin B 2006. Length-Weight Relationships for 47 Coastal Fish Species from the Northern Aegean Sea, Turkey. Journal of Applied Ichthyology, 22: 274-278.

La Mesa M, Colella S, Giannetti G, Arneri E. 2008. Age and Growth of Brown Meagre Sciaena umbra (Sciaenidae) in the Adriatic Sea. Aquatic Living Resources, 21: 153-161.

Mater S, Kaya M, Bilecenoğlu M 2009. Marine Fishes of Turkey (4th press), Ege University Fisheries Faculty Publishings, No. 68, İzmir (In Turkish).

Moutopoulos DK, Stergiou KI 2002. Length-Weight and Length-Length Relationships of Fish Species from the Aegean Sea (Greece). Journal of Applied Ichthyology, 18: 200-203.

Navarro MR, Villamor B, Myklevoll S, Gil J, Abaunza P, Canoura J 2012. Maximum Size of Atlantic mackerel (Scomber scombrus) and Atlantic chub mackerel (Scomber colias) in the Northeast Atlantic. Cybium 36: 406-408.

Özaydın O, Bilecenoğlu M, Kaya M 2000. Age and Growth of the Curled Picarel Centracanthus cirrus Rafinesque, 1810 (Osteichthyes: Centracanthidae) in Northern Cyprus, Eastern Mediterranean Sea. Acta Adriatica 41: 35-42.
Özekinci U, Cengiz Ö, İşmen A, Altınağaç U, Ayaz A 2009. Length-Weight Relationships of Thirteen Flatfishes (Pisces: Pleuronectiformes) from Saroz Bay (North Aegean Sea, Turkey). Journal Animal and Veterinary Advences, 8: 1800-1801.

Petrakis D, Stergiou KI 1995. Weight-Length Relationships for 33 Fish Species in Greek waters. Fisheries Research, 21: 465-469.

Richter H, Lückstädt C, Focken U, Becker K 2000. An Improved Procedure to Assess Fish Condition on the Basis of Length-Weight Relationships. Archive of Fishery and Marine Research, 48: 255-264.

Ricker WE 1975. Computation and Interpretation of Biological Statistics of Fish Populations. Journal of the Fisheries Research Board of Canada, 191: 1382.

Safran P 1992. Theoretical Analysis of the WeightLength Relationships in Fish Juveniles. Marine Biology, 112: 545-551.

Tokat E, Sayın E 2007. Water Masses Influencing the Hydrographic Properties of Saros Bay. Rapp Comm Int Mer Medit, 38: 205.

Torres MA, Ramos F, Sobrino I 2012. Length-Weight Relationships of 76 Fish Species from the Gulf of Cadiz (SW Spain). Fisheries Research, 127-128: 171-175.

Verdiell-Cubedo D, Oliva-Paterna FJ, Torralva M 2006. Length-Weight Relationships for 22 Fish Species of the Mar Menor Coastal Lagoon (western Mediterranean Sea). Journal of Applied Ichthyology, 22: 293-294.

Wootton RJ 1998. Ecology of Teleost Fishes. 2nd Edition, Kluwer Academic, London. 